\title{
Breast Surgery can be Performed Safely During the COVID-19 Pandemic: A Retrospective Single-Center Analysis
}

\author{
(1) Akif Enes Arikan1,2, (1) Halil Kara2,3, (D) Onur Dülgeroğlu2,3, (1) Cihan Uras1,2 \\ ${ }^{1}$ Department of General Surgery, Acibadem Mehmet Ali Aydinlar University School of Medicine, İstanbul, Turkey \\ 2Acibadem Mehmet Ali Aydinlar University Research Institute of Senology, İstanbul, Turkey \\ ${ }_{3}^{3}$ ccibadem Mehmet Ali Aydinlar University Vocational School of Health Sciences, İstanbul, Turkey
}

\begin{abstract}
Introduction: The Coronavirus disease-2019 (COVID-19) outbreak has affected the diagnosis and treatment of various diseases including breast cancer. This study aimed to investigate whether breast surgery can be performed safely during the COVID-19 pandemic.

Methods: Patients who underwent surgery for breast cancer or suspicious breast lesions in the pre-pandemic, first wave, and second wave periods of the pandemic were evaluated retrospectively.

Results: Data of 220 patients who underwent breast surgery were analyzed. No significant difference was found between the prepandemic, first wave, and second wave periods of the COVID-19 pandemic in terms of patient characteristics, complications, types of complication, Clavien-Dindo classification of complications, and complications requiring intervention. No COVID-19 related complication was also observed.

Conclusion: Breast surgery can be performed safely in the COVID-19 pandemic. For safe surgery, appropriate precautionary measures against COVID-19 and COVID-19 screening should be initiated. COVID-19-free surgical pathway is also important for safe surgery. With the continuation of surgeries, fear of upstaging, subsequent requirement of more aggressive treatment for tumors, and postpandemic overload can be prevented.
\end{abstract}

Keywords: COVID-19, breast, breast surgery, complication

\section{Introduction}

The Coronavirus disease-2019 (COVID-19) outbreak caused by severe acute respiratory syndrome-coronavirus-2 (SARS-CoV-2) has spread rapidly worldwide since December 2019 and declared as a pandemic by the World Health Organization (WHO) on March 11, 2020 (1,2). With the rapid spread of the infection, various quarantine measures had to be taken.

Since the first case on March 11, 2020, additional measures were taken well and quarantine protocol were also initiated in Turkey $(1,2)$ : i.e., declaration of both state and private hospitals as pandemic hospitals, emphasizing the importance of social isolation, encouraging social isolation, and implementing curfews including people aged $\geq 65$ years or those with comorbidities. A circular was issued by the Ministry of Health of Turkey to stop surgeries other than emergency and cancer surgeries. During this period, it was unknown how COVID-19 would affect the surgical results. Suggestions related to COVID-19 management have been reported for many malignancies, including breast cancer (1,3-5). During the pandemic, the safety of surgeries was questioned, and some authors expressed an opinion that surgeries should be delayed, if possible. On the contrary, the postponement of surgeries and a decrease in hospital admissions lead to a fear of upstaging and therefore a wider treatment requirement with enlarging tumors. However, over time, studies have started reporting that safe surgery can be performed in the pandemic period have. Thus, this study aimed to investigate whether safe breast surgery can be performed during the COVID-19 pandemic.

\section{Methods}

Based on the daily number of patients with COVID-19 taken from the website of the Ministry of Health of Turkey, the first wave of the COVID-19 pandemic was considered between April 1, 2020, and May 15, 2010, and the second wave was between November 15, 2020, and December 31, 2020 (6). Although the first case in Turkey was seen on March 11, 2020, the period between March 11 and March 31, 2020, was not included in the first wave because of the small number of COVID-19 cases.

In this study, patients who underwent upfront surgery for breast cancer or suspicious breast lesions or surgery after neoadjuvant chemotherapy 
(NACT) for breast cancer in the first and second wave of the pandemic were evaluated retrospectively. As the control group, patients who underwent surgery between December 15, 2019, and March 15, 2020, were evaluated retrospectively. The study was conducted in Acibadem Mehmet Ali Aydinlar University Research Institute of Senology (RISA).

Age, sex, tumor side, hospital stay duration, tumor type (benign, invasive, or non-invasive), type of initial treatment (upfront surgery or NACT), and number and type of surgery to the breast and axilla of each patient were recorded. Procedures were evaluated separately as upfront surgery and surgeries performed after NACT. Procedures were first evaluated in three groups as excisional biopsies, breast-conserving surgeries (BCS), and mastectomies. Subsequently, mastectomies were evaluated in separate groups as simple, skin-sparing, and nipplesparing mastectomies (NSM). Excisional biopsies included surgeries performed for suspicious lesions in which only the lesion is excised. BCS included surgeries in which the entire breast was not removed. Simple mastectomy included mastectomies in which the nipple and skin were removed without simultaneous reconstruction. Skin-sparing mastectomies included mastectomies in which the nipple is removed, but simultaneous reconstruction is performed while preserving the breast skin. NSM included mastectomies in which both the nipple and breast skin were preserved, and simultaneous reconstructions were performed. Reconstructions were examined in two groups as reconstructions with silicone implants or autologous tissues. Axillary surgeries were examined in two groups as sentinel lymph node biopsy and axillary lymph node dissection.

Complications were evaluated according to the type of surgery to both the breast and axilla and whether they developed after upfront surgery or surgeries after NACT. The severity of the complications was evaluated according to the Clavien-Dindo classification (7). If there was more than one complication in the same patient, the patient was evaluated according to the more serious complication based on the Clavien-Dindo classification.

With the declaration of the pandemic in Turkey, RISA-affiliated hospitals were declared as pandemic hospitals. Multidisciplinary tumor meetings were conducted with the participation of doctors on-site by restricting the participation of nurses, secretaries, and allied health professionals. All patients were discussed and treatment decisions were made at the multidisciplinary tumor meetings as in the pre-pandemic period. During the pandemic, all surgeries were performed in COVID-19-free operating rooms and patients were hospitalized in COVID-19-free wards. During the pandemic period, low-dose thoracic computed tomography was taken for each patient scheduled for surgery until June 1, 2020. After this time, reverse-transcriptase polymerase chain reaction (PCR) testing for SARSCoV-2 was used in accordance with the European guidelines (8). Surgeries of patients with positive PCR for COVID-19 were delayed for 4 weeks until having negative PCR results and disappearance of symptoms. The algorithm applied to patients before surgery is shown in Figure 1.

Patients who did not have any postoperative complaints were called for a follow-up on the $10^{\text {th }}$ postoperative day to evaluate the wound site and to inform the patient about the postoperative treatment protocols. If the patient had complaints, the patient was assessed by phone. If the

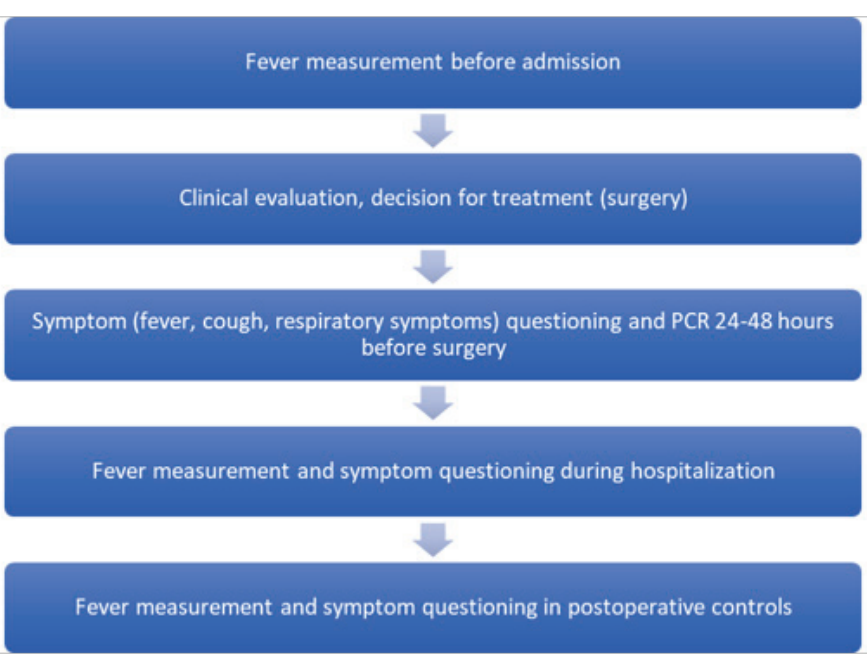

Figure 1. Algorithm applied to patients before surgery for COVID-19 screening

COVID-19: Coronavirus disease-2019, PCR: polymerase chain reaction

complication was considered as at least class 2 according to the ClavienDindo classification, an in-hospital appointment was planned.

\section{Ethical approval}

Ethics committee approval from the Ministry of Health of Turkey was taken (file no: Akif Enes ARIKAN-2021-03-20T12_50_30) and from the Ethics Committee of Acibadem Mehmet Ali Aydinlar University (approval number: 2021-06/25, date: 24.03.2021).

\section{Statistical Analysis}

Study data were collected and managed using research electronic data capture (REDCap) tools hosted at the Acibadem Mehmet Ali Aydinlar University (9). All statistical analyses were performed using SPSS 25.0 (IBM Corp., Armonk, NY, USA). A p-value $<0.05$ was considered significant. Analysis of variance was performed for continuous variables, and a chi-square or Fisher's exact test was used for categorical variables. Continuous data are reported as mean \pm standard deviation.

\section{Results}

Data of 220 patients who underwent breast surgery during the first and second wave of the COVID-19 pandemic and of the control group between December 15, 2019, and March 15, 2021, were retrospectively analyzed.

The COVID-19 PCR test was positive in two patients with cough during the preoperative period. Preoperative PCR test was positive for two patients without symptoms. The surgeries of these patients with positive PCR were delayed for 4 weeks after the symptoms resolved and the PCR results became negative. No COVID-19 case was detected in the first 30day postoperatively in any of the patients, including patients with PCRpositive results preoperatively, and no COVID-19-related complications were observed. In addition, COVID-19 was not detected in healthcare personnel participating in breast surgeries. COVID-19 developed in two surgeons from the surgical team and the sources of infections were their family members. 
Table 1. Characteristics of the patients

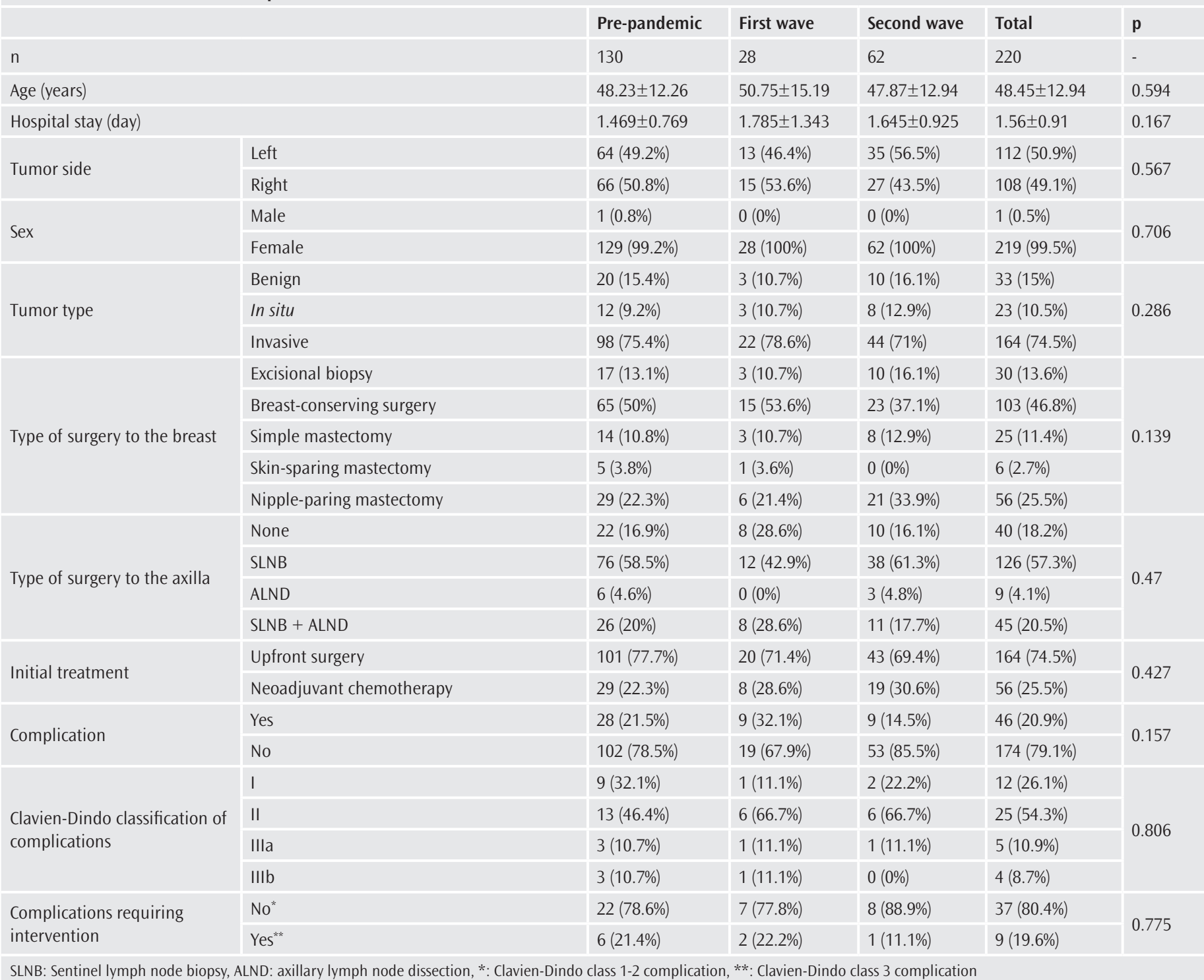

Table 2. Type and timing of complications after breast surgery

\begin{tabular}{|l|l|l|}
\hline Complications & Period \\
\hline Fibrosis & Early (<30 days) & Late (>30 days) \\
\hline Hematoma & - & $1(6.3 \%)$ \\
\hline Lymphedema & $2(5.6 \%)$ & - \\
\hline Necrosis (full thickness) & - & $3(18.8 \%)$ \\
\hline Ischemia & $1(2.8 \%)$ & - \\
\hline Capsule formation & $2(5.6 \%)$ & - \\
\hline Prosthesis rejection & - & $3(18.8 \%)$ \\
\hline Seroma & - & $3(18.8 \%)$ \\
\hline Wound infection & $21(58.3 \%)$ & $5(31.3 \%)$ \\
\hline Fever & $4(11.1 \%)$ & - \\
\hline Postoperative nausea & $2(5.6 \%)$ & - \\
\hline Rotation of implant & $4(11.1 \%)$ & - \\
\hline & - & $1(6.3 \%)$ \\
\hline
\end{tabular}

No significant difference was found between the pre-pandemic, first wave, and second wave periods in terms of age, sex, hospital stay, tumor site, tumor type (benign/in situ/invasive), type of initial treatment, and type of surgery to the breast and axilla. The characteristics of the patients are shown in Table 1.

The types and timing (early/late period) of the complications are shown in Table 2. Clavien-Dindo classification of complications according to the type of initial treatment and pandemic period is shown in Table 3.

No significant difference was noted between the pre-pandemic, first wave, and second wave periods in terms of complication development, complication type, Clavien-Dindo classification of complications, and complications requiring intervention.

\section{Discussion}

In this single-center study of 220 patients, no complications related to COVID-19 were detected in breast surgeries performed in the first and second waves of the pandemic period. In addition, the number 
Table 3. Clavien-Dindo classification of complications according to the type of initial treatment and pandemic period

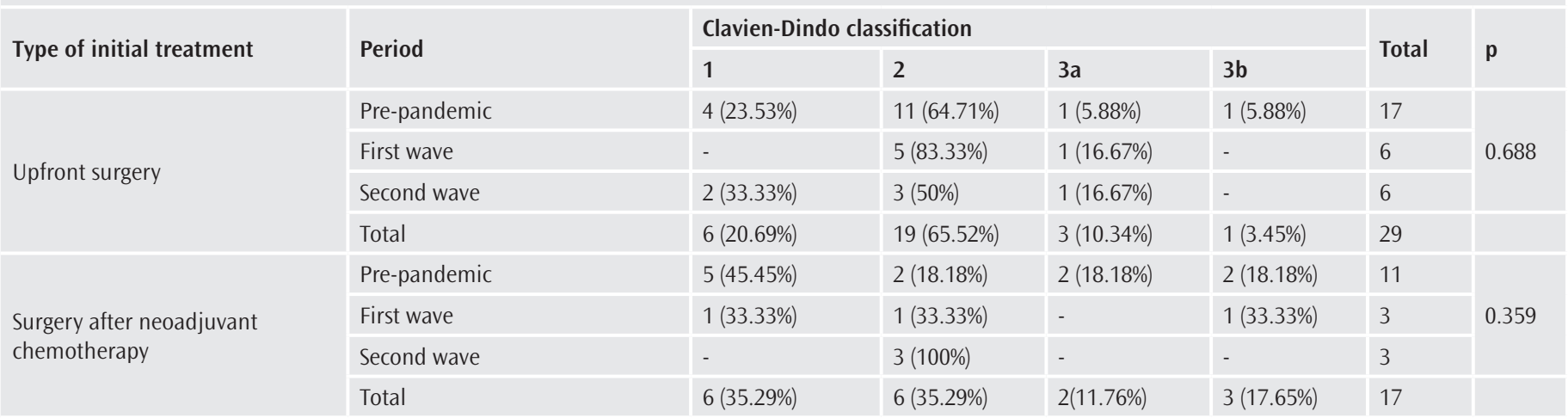

of complications did not increase because of breast surgery. Our study shows that with appropriate precautions, breast surgery can be performed safely in the pandemic period.

Following the announcement of the COVID-19 pandemic by the WHO, measures have been taken in many countries. Like other countries, the government of Turkey emphasized the importance of social isolation and directed people to practice social isolation. Curfews were implemented for patients aged $>65$ or for those with comorbidities. Private and public hospitals were declared as pandemic hospitals, and a circular was issued by the Ministry of Health of Turkey to stop surgeries other than emergency and cancer surgeries.

The safety of surgeries was questioned in the early period of the pandemic. Studies in this period had reported high mortality and morbidity associated with surgeries, especially in patients with COVID-19 $(4,10,11)$. In the international cohort study by the COVIDSurg group covering 1,128 patients who underwent surgery between January 1 and March 31, and 294 of whom were diagnosed with COVID preoperatively, the 30 -day mortality rate was $23.8 \%$ (268 of 1,128) (10). Pulmonary complications occurred in 577 (51.2\%) of 1,128 patients, and the 30day mortality rate in these patients was 38.0\% (219 of 577), accounting for $81.7 \%$ (219 of 268) of all deaths. Many authors have recommended postponement of surgeries if possible in the early period of the COVID-19 pandemic due to reasons such as the effects of COVID-19 on mortality and morbidity and the allocation of hospital beds and sources to patients with COVID-19 (1,3-5). Moreover, since COVID-19 was seen more in healthcare workers in the early pandemic period, ensuring the protection of healthcare workers had also been effective on this decision $(12,13)$.

However, studies reporting that safe surgery can be performed in the pandemic period have started to emerge (14-23). These studies have reported that complications due to surgery do not increase during the pandemic and that COVID-19-associated complications are not high. In the present study, as there was no increase in complications during the pandemic period, no COVID-19-related complications were observed.

Although the risks associated with COVID-19 are high in patients with malignancy in the early pandemic period, there are publications that show breast cancer does not pose a high risk for COVID-19 $(24,25)$. In the study involving 279 patients who received chemotherapy, 92 of which had breast cancer, between January 19 and April 2020, more complications were found in hematologic cancers compared than in solid tumors (24). The author reports that treatment can be applied by taking precautions in solid cancers such as breast cancer. Similarly, Zhang et al. (25) compared 35 patients with COVID-19 and breast cancer and 55 patients with COVID-19 without breast cancer and 81 patients with COVID-19 and cancer other than breast cancer as controls. They reported no differences in disease severity and outcomes between patients with COVID-19 and breast cancer and common patients with COVID-19. Moreover, the clinical characteristics of patients with breast cancer were milder than those with other types of cancers. In line with these studies, we can state that breast cancer does not pose a high risk for COVID-19, considering the short duration of hospitalizations because of breast surgery (26).

Ji et al. (16) evaluated a total of 621 patients, 141 of whom underwent breast-endocrine surgery, who underwent elective cancer surgery between 01.02.2020 and April 27, 2020. While PCR was performed in only symptomatic cases $(n=40,6.4 \%)$, COVID-19 infection was not detected in any patients postoperatively.

One of the most important studies regarding safe surgery during the COVID-19 pandemic is the study by the COVIDSurg group, which includes 9,171 patients, of which 2,140 had undergone breast surgery (17). In total, 2,481 patients received surgery following the COVID19-free surgical pathway (i.e., complete segregation of the operating theater, critical care, and inpatient ward areas). COVID-19-free surgical pathways were followed for younger patients with less comorbidities. The postoperative SARS-CoV-2 infection rate was also lower in COVID-19free surgical pathways. Pulmonary complication rates were lower with COVID-19-free surgical pathways (2.2\% vs $4.9 \%)$. Therefore, they suggest that dedicated COVID-19-free surgical pathways should be established to provide safe elective cancer surgery in the current and before future outbreaks of SARS-CoV-2.

Another important study of Kane et al. (18) showed that elective surgeries can be performed under appropriate conditions; the study involved 557 patients between March 29 and June 12, 2020. Patients without COVID-19 symptoms were screened by oronasal swab and chest imaging (chest X-ray or computed tomography if aged $\geq 18$ years) and preceded to surgery if negative. While $13(2.4 \%)$ patients tested positive during screening, 7 (1.4\%) tested positive for COVID-19 [1.4\%, 95\% confidence interval (CI): 0.7-2.8\%] with one COVID-19-related death $(0.2 \%, 95 \% \mathrm{Cl}$ : $0.0-1.1 \%)$ within 30 days. 
Another issue related to the postponement of surgeries during the pandemic is the overload problem that may occur after the pandemic. The COVIDSurg group performed an estimation using a Bayesian $\beta$-regression model for 12 -week cancellation in 190 countries (27). The best estimate was that 28,404,603 operations would be cancelled or postponed. If countries increased their normal surgical volume by $20 \%$ after the pandemic, a median of 45 weeks is needed to clear the backlog of operations resulting from the COVID-19 disruption. The rapid decrease in the number of patients in the early period of the pandemic supports this estimation. However, the number of operations has increased after the first wave because of reasons such as the adaptation of the clinic and patients, fluctuating course of the pandemic, and application of COVID-free protection methods throughout the country. To prevent this scenario, which the COVIDSurg group estimates, appropriate measures should be taken and the surgeries should continue. Therefore, hospitals can be made available for surgery or not every hospital is declared a pandemic, and some can be spared.

In the COVID-19 pandemic or similar pandemics that may occur in the future, precautions should be taken from the first diagnosis of the patient to the end of the treatment so that the surgeries can continue, treatments are not delayed, and post-pandemic overload can be prevented. These measures should include the following:

- Hospitals with COVID-19-free surgical pathway should be organized, and these hospitals should not be declared as pandemic hospitals and should be spared for oncological treatments.

- Patients should be questioned in terms of COVID-19 related complaints at the time of admission and follow-up.

- Symptom screening and suspicious travel questioning should be conducted at the entrance of the hospital, and patients with suspicious symptoms should be directed to the COVID-19 clinic.

- At the beginning of the treatment, in line with the guidelines of the institutions, PCR, chest radiography, or low-dose thorax CT should be performed for COVID-19.

- In the hospital, patients should wear a mask without valve.

- The number of companions should be reduced.

- To provide a social distance between patients, the space between the seats should be widened and daily magazine or tabloid magazines should be removed.

- The use of personal protective equipment by all healthcare professionals is mandatory.

- Healthcare professionals and patients should pay attention to hand hygiene.

- The number of healthcare personnel and patients in all areas within the hospital should be minimized.

- Movements of patients and healthcare personnel between departments should be minimized.

- In areas where healthcare personnel gather, such as dining halls, the sitting areas should be made sparse. Meals can be prepared in disposable containers, and the healthcare personnel can eat in the department where they work or the meals can be sent to the departments to reduce contacts between departments.

- In operating rooms, a minimum number of medical personnel should be employed.

- In case of delay or delay in elective breast cancer surgery, a metallic marker should be placed in every patient who has an image-guided biopsy if it does not contain microcalcification.

- Telemedicine can be used to reduce the number of hospital admissions (28).

\section{Conclusion}

Breast surgery can be performed safely during the COVID-19 pandemic. For safe surgery, appropriate precautions against COVID-19 should be taken and COVID-19 screening should be performed. COVID-19-free surgical pathway (i.e., complete segregation of the operating theater, critical care, and inpatient ward areas) is also important for a safe surgery. With the continuation of surgeries, our method can prevent the fear of upstaging and therefore requirement of more aggressive treatment for tumors and post-pandemic overload.

Ethics Committee Approval: Ethics committee approval from the Ministry of Health of Turkey was taken (file no: Akif Enes ARIKAN-202103-20T12_50_30) and from the Ethics Committee of Acibadem Mehmet Ali Aydinlar University (approval number: 2021-06/25, date: 24.03.2021).

Informed Consent: Retrospective study.

Peer-review: Externally and internally peer-reviewed.

Authorship Contributions: Surgical and Medical Practices - O.D., C.U.; Concept - A.E.A., H.K., O.D., C.U.; Design - A.E.A., H.K., O.D., C.U.; Data Collection or Processing - O.D., C.U.; Analysis or Interpretation - A.E.A., H.K.; Literature Search - H.K., O.D.; Writing - A.E.A., H.K., O.D., C.U.

Conflict of Interest: No conflict of interest was declared by the authors.

Financial Disclosure: The authors declared that this study received no financial support.

\section{References}

1. Cakmak GK, Ozmen V. Sars-CoV-2 (COVID-19) Outbreak and Breast Cancer Surgery in Turkey. Eur J Breast Health 2020; 16: 83-5.

2. Citgez B, Yigit B, Capkinoglu E, Yetkin SG. Management of Breast Cancer during the COVID-19 Pandemic. Sisli Etfal Hastan Tip Bul 2020; 54: 132-5.

3. Sezer A, Cicin I, Karadeniz Cakmak G, Ozkan Gurdal S, Basaran G, Oyan $B$, et al. Turkish national consensus on breast cancer management during temporary state of emergency due to COVID-19 outbreak. Turk J Surg 2020; 36: 147-63.

4. Dietz JR, Moran MS, Isakoff SJ, Kurtzman SH, Willey SC, Burstein HJ, et al. Recommendations for prioritization, treatment, and triage of breast cancer patients during the COVID-19 pandemic. the COVID-19 
pandemic breast cancer consortium. Breast Cancer Res Treat 2020; 181: 487-97.

5. Society of Surgical Oncology. Resource for Management Options of Breast Cancer During COVID-19 2020 [updated March 30, 2020]. Available from: https://www.surgonc.org/wp-content/ uploads/2020/03/Breast-Resource-during-COVID-19-3.30.20.pdf

6. Republic of Turkey Ministry of Health. General Coronavirus Table 2021 [cited 2021, April 1]. Available from: https://covid19.saglik.gov. tr/TR-66935/genel-koronavirus-tablosu.html

7. Dindo D, Demartines N, Clavien PA. Classification of surgical complications: a new proposal with evaluation in a cohort of 6336 patients and results of a survey. Ann Surg 2004; 240: 205-13.

8. Corman VM, Landt O, Kaiser M, Molenkamp R, Meijer A, Chu DK, et al. Detection of 2019 novel coronavirus (2019-nCoV) by real-time RTPCR. Euro Surveill 2020; 25: 2000045.

9. Harris PA, Taylor R, Minor BL, Elliott V, Fernandez M, O'Neal L, et al. The REDCap consortium: Building an international community of software platform partners. J Biomed Inform 2019; 95: 103208.

10. COVIDSurg Collaborative. Mortality and pulmonary complications in patients undergoing surgery with perioperative SARS-CoV-2 infection: an international cohort study. Lancet 2020; 396: 27-38.

11. Abate SM, Mantefardo B, Basu B. Postoperative mortality among surgical patients with COVID-19: a systematic review and metaanalysis. Patient Saf Surg 2020; 14: 37.

12. Shimpi T, Kulkarni S, Bukhanov K, Fleming R, Scaranelo A, Ghai S, et al. Tailored breast imaging during the first wave and preparedness for the second wave of COVID-19 pandemic. Eur J Radiol Open 2020; 7: 100265 .

13. Wei JT, Liu ZD, Fan ZW, Zhao L, Cao WC. Epidemiology of and Risk Factors for COVID-19 Infection among Health Care Workers: A MultiCentre Comparative Study. Int J Environ Res Public Health 2020; 17 : 7149 .

14. Fregatti P, Gipponi M, Giacchino M, Sparavigna M, Murelli F, Toni ML, et al. Breast Cancer Surgery During the COVID-19 Pandemic: An Observational Clinical Study of the Breast Surgery Clinic at Ospedale Policlinico San Martino - Genoa, Italy. In Vivo 2020; 34: 1667-73.

15. Filipe MD, van Deukeren D, Kip M, Doeksen A, Pronk A, Verheijen PM, et al. Effect of the COVID-19 pandemic on surgical breast cancer care in the Netherlands: A Multicenter Retrospective Cohort Study. Clin Breast Cancer 2020; 20: 454-61.

16. Ji C, Singh K, Luther AZ, Agrawal A. Is elective cancer surgery safe during the COVID-19 pandemic? World J Surg 2020; 44: 3207-11.
17. Glasbey JC, Nepogodiev D, Simoes JFF, Omar O, Li E, Venn ML, et al. Elective Cancer Surgery in COVID-19-Free Surgical Pathways During the SARS-CoV-2 Pandemic: An International, Multicenter, Comparative Cohort Study. J Clin Oncol 2021; 39: 66-78.

18. Kane AD, Paterson J, Pokhrel S, Berry SK, Monkhouse D, Brand JW, et al. Peri-operative COVID-19 infection in urgent elective surgery during a pandemic surge period: a retrospective observational cohort study. Anaesthesia 2020; 75: 1596-604.

19. Romics L, Doughty J, Stallard S, Mansell J, Blackhall V, Lannigan A, et al. A prospective cohort study of the safety of breast cancer surgery during COVID-19 pandemic in the West of Scotland. Breast 2021; 55: $1-6$.

20. Abdalla AS, Asaad A, Fisher R, Clayton G, Syed A, Barron M, et al. The Challenge of COVID-19: The Biological Characteristics and Outcomes in a Series of 130 Breast Cancer Patients Operated on During the Pandemic. Chirurgia (Bucur) 2020; 115: 458-68.

21. Maclnnes EG, Piper J, Tait C, Waterworth A, Achuthan R, Hogan B, et al. Breast Cancer Surgery During the COVID-19 Pandemic Peak in the UK: Operative Outcomes. Cureus 2020; 12: e9280.

22. Vanni G, Pellicciaro M, Materazzo M, Dauri M, D’Angelillo R M, Buonomo C, et al. Awake breast cancer surgery: strategy in the beginning of COVID-19 emergency. Breast Cancer 2021; 28: 137-44.

23. Simoes J, Bhangu A, CovidSurg Collaborative. Should we be restarting elective surgery? Anaesthesia 2020; 75: 1563-5.

24. Aznab M. Evaluation of COVID 19 infection in 279 cancer patients treated during a 90-day period in 2020 pandemic. Int J Clin Oncol 2020; 25: 1581-6.

25. Zhang B, Xie R, Hubert SM, Yu Y, Zhang Y, Lei X, et al. Characteristics and Outcomes of 35 Breast Cancer Patients Infected With COVID-19. Front Oncol 2020; 10: 570130.

26. Marla S, McMillan DC, Stallard S. Factors influencing postoperative length of hospital stay after breast cancer surgery. Breast 2013; 22: 289-94.

27. COVIDSurg Collaborative. Elective surgery cancellations due to the COVID-19 pandemic: global predictive modelling to inform surgical recovery plans. Br J Surg 2020; 107: 1440-9.

28. Zhu C, Williamson J, Lin A, Bush K, Hakim A, Upadhyaya K, et al. Implications for Telemedicine for Surgery Patients After COVID-19: Survey of Patient and Provider Experiences. Am Surg 2020; 86: 90715. 